\title{
La tendencia del desarrollo sustentable en la educación en ingeniería en México
}

\section{The tendency of the sustainable development of the Mexico's engineering study program}

\author{
MARCOS-SORIANO, José Antonio†, CRUZ-GÓMEZ, Marco Antonio*, MEJÍA-PÉREZ, José \\ Alfredo y ESPINOSA-CARRASCO, María del Rosario \\ Benemérita Universidad Autónoma de Puebla; Facultad de Ingeniería, Grupo de Tribología y Transporte, Cuerpo \\ Académico 189 (Prevención de Desastres and Desarrollo Sustentable, Tribología, BUAP), Edificio de Posgrado, Primer \\ nivel, Cubículo Núm. 16, Blvd. Valsequillo esq. Av. San Claudio, Ciudad Universitaria, Col. San Manuel, CP. 72570, \\ Puebla, México
}

ID $1^{\mathrm{er}}$ Autor: José Antonio, Marcos-Soriano / ORC ID: 0000-0002-8653-7663, Researcher ID Thomson: AAP-27742020, CVU CONACYT ID: 1067102

ID $1^{\mathrm{er}}$ Coautor: Marco Antonio, Cruz-Gómez / ORC ID: 0000-0003-1091-8133, Researcher ID Thomson: S-3098-2018, CVU CONACYT ID: 349626

ID $2^{\text {do }}$ Coautor: José Alfredo, Mejía-Pérez / ORC ID: 0000-0002-4090-8828, Researcher ID Thomson: G-3354-2019

ID $3^{\text {er }}$ Coautor: María del Rosario, Espinosa-Carrasco / ORC ID: 0000-0002-5094-2800, Researcher ID Thomson: AAP2965-2020, CVU CONACYT ID: 1018747

DOI: $10.35429 / J U S D .2020 .18 .6 .10 .17$

Recibido: 15 de Enero, 2020; Aceptado 30 de Junio, 2020

\section{Resumen}

El artículo discute la implementación de constantes actualizaciones en la gestión y programas de estudio de las Universidades en México, con el objetivo de avanzar en una Industria cambiante que requiera ingenieros que puedan adaptarse a las tendencias que conllevan a una relación entre Instituciones formadoras de ingenieros e Industrias con intención de crear proyectos sustentables y conseguir el desarrollo técnico y tecnológico al servicio de la sociedad. El artículo presenta un escenario en el cual los estudiantes de ingeniería y las empresas consiguen el equilibrio de una ideología basada en el compromiso de desarrollar o adaptar proyectos enfocados a construir un entorno sustentable de modernización regional. Numerosos estudios indican la necesidad de instruir un desarrollo capaz de orientar a la comunidad de estudiantes de ingeniería a crecer en armonía con la sociedad y el medio ambiente.

\begin{abstract}
The article discusses the implementation of constant updates in the management of the National College study programs, with the main goal to advance in a constantly changing Industry sector that requires engineers who could adapt themselves to the tendencies that build solid relations between forming Institutions of engineers (colleges) and the Industry sector, with the purpose of creating sustainable projects obtain the technical and technological development to help our society. The article presents a stage in which the Engineering students and the companies obtain the firmness of an ideology based on the commitment to develop or to adapt projects focused to constructing a sustainable environment that would impulse the technology in the local region, modernizing it. Many studies show the necessity of teaching and transmitting a development capable of guiding the Engineering student's community to the path of a harmonic evolution with the society and the environment.
\end{abstract}

Tendencies, Engineering, sustainable environment

\footnotetext{
*Correspondencia al autor (Correo electrónico: mangcruz@live.com)

$\dagger$ Investigador contribuyendo como primer autor
} 


\section{Introducción}

Una sociedad sustentable es capaz de subsistir y prosperar indefinidamente, encontrando el equilibrio entre los recursos naturales, el desarrollo económico y la calidad de vida en lo que respecta a las condiciones que promueven el sostenimiento, crecimiento y prosperidad humana individual y colectiva. En cuanto a la autodeterminación o autonomía de los estudiantes se puede observar que su interés de estudiar una disciplina se impulsa fomentando un sistema de estudio atractivo enfocado a su motivación y resiliencia para mantener una prospectiva positiva de un pensamiento lateral. Las Universidades son tanto el punto de partida del progreso exitoso de la humanidad a lo largo del tiempo como la estructura que continúa avanzando, modificándose muchas veces hasta llegar a una etapa de madurez demostrando estabilidad (Glover \& Peters, 2013; Mulder et al., 2013; Pappas, et al., 2013).

El principal problema de la educación en ingeniería es limitarse a conceptos y procesos que dan respuesta a las necesidades de las Industrias sin mirar y evaluar los problemas e interacciones medioambientales. Es por ello que la noción de sustentabilidad debe ser integrada a las Universidades. (Staniskis \& Katiliute, 2015; Ortega, 2020).

Son cuatro contextos que facilitan la enseñanza de sustentabilidad, el primero implica a los individuos, la relación entre grupos sociales, la familia, comportamiento colectivo, clase social, raza y etnia, salud, educación y el papel de las Instituciones en la sociedad. Esto incluye factores culturales, valores, actitudes, creencias, comportamientos y prácticas sociales que caracterizan el conocimiento y el comportamiento humano. El segundo es el diseño orientado a la ingeniería de procesos, productos y estructuras que tiende a producir un efecto negativo, uno neutro o un efecto benigno en todos los sistemas ambientales. El tercer contexto va más allá de las políticas económicas y los procesos de fabricación, está directamente relacionado con la opción de un desarrollo eficiente, rentable y sustentable que pueden influir en la salud económica y el perfil de las comunidades, incluyendo el nivel de vida, el estado comercial existente, empleo y el rol productivo de la corporación en la vida de sus empleados.
El siguiente abarca una amplia variedad de factores dependientes relacionados con el diseño y fabricación de productos con base en la investigación científica y tecnología eficiente de uso duradero que cumplan su funcionamiento (Idem).

El avance y manejo de las tecnologías para aumentar la productividad es el factor principal que está adoptando nuevos roles para la aplicación del conocimiento científico y proporcionar a la sociedad aquellas cosas que son necesarias o deseadas. Numerosos ejemplos de tecnología se encuentran en nuestra vida cotidiana, ya sea directamente o no. En consecuencia, las Universidades requieren formar ingenieros comprometidos con un trabajo responsable con la capacidad de responder ante los problemas que requieran una solución a corto plazo. De tal manera que cada materia que los alumnos cursan durante su estancia en la Universidad pueda ser aplicado a dichos problemas con iniciativa de ideas innovadoras (Sonmez, 2014; Abdul, 2016).

El resto del documento describe las propuestas que beneficiarán a una sociedad dirigida a un crecimiento seguro con la capacidad de eliminar o disminuir situaciones no deseadas en el propio sistema. En consecuencia, se destacan los apartados de la sustentabilidad en la educación, la gestión del proceso hacia la calidad y para terminar la vinculación de la ingeniería en la Industria.

\section{Sustentabilidad en la educación}

La educación de calidad en ciencias e ingeniería es un reto de prioridad, pero también es la clave para la innovación tecnológica que impulsa el desarrollo económico nacional. El plan de estudios es la parte más importante de los sistemas de educación en ingeniería, ya que es la base del conocimiento que poseen los graduados para su empleo inmediato. Este debe garantizar que la estructura de su curso responda la del mercado y al mismo tiempo atender las necesidades de la comunidad. Con respecto al tiempo de implementación de un programa de estudios para lograr el desarrollo sustentable de manera inteligente se deben planear estrategias de integración a corto, mediano y largo plazo que adecuen el contenido teórico y práctico a un entorno dinámico en el país (Chowdhury et al., 2013; Mujumdar, 2014; Momete, 2015). 
Los programas de estudio deben prestar atención a dos paradigmas cruciales para su elaboración. Por un lado, se busca crear conciencia para preservar el medio ambiente debido a los bastos procesos industriales por medio del análisis en los sistemas ambientales y por otro lado se pretende aprovechar las tecnologías presentes para medir y comprender fenómenos naturales y el dinamismo social mediante la gestión del cambio y la innovación. No hay duda de que los planes de estudio deben actualizarse continuamente a medida que se añaden nuevos conocimientos, ya que los ingenieros de hoy necesitan más que solo una sólida formación técnica para tener éxito. Las Instituciones con estas actualizaciones aspiran a convertirse en generaciones de innovación, nuevos conocimientos y tecnología (Chowdhury et al., 2013; Derrick, 2013; Mulder, 2017).

El alcance del plan de estudios puede variar desde nuevo material en un módulo existente hasta uno completamente nuevo. Incluso se ha discutido incorporar algunos juegos que pueden usarse para integrar y capacitar a los estudiantes para pensar y actuar de nuevas formas y más sustentable pero aún falta mucho por hacer. Pese a que la integración del desarrollo sustentable es lento, limitado y poco sistemático y que los graduados son desequilibrados, sobreespecializados y monodisciplinarios es necesario un esfuerzo adicional para supervisar y evaluar la profundidad de los programas de estudio (Lozano y Watson, 2013; Thurer et al., 2018).

Un aspecto importante para el estudio en la literatura sobre lo sustentable es el tiempo, con algunas excepciones notables, se trata del pasado, el presente y el futuro. Derrick sugiere el concepto de una orientación pasada que describe patrones de desigualdad y las consecuencias no deseadas de las tecnologías. Una orientación actual que mide lo que tenemos, que es gran parte el resultado de acciones pasadas. Una orientación futura que cuestiona los indicadores y factores para un nivel de consumo razonable a nivel individual y nacional para lograr o mantener la sustentabilidad (Idem; Aliaga \& Luna, 2020).
Los estudiantes de ingeniería deben tener vínculos claros con la educación ambiental, promover y mejorar la calidad de una educación de por vida. Sin embargo, este proceso de aprendizaje conduce a los alumnos a pensamientos críticos independientes, así como comportamientos de cambio de hábitos que les facilite asumir los entornos que se les presenten. Ya que los escenarios son inciertos requiere de personas capaces de analizar y abordar nuevos desafíos sistemáticamente. En este contexto fomentar la capacidad de reflexión crítica es crucial para implementar estrategias orientadas a grandes problemas ambientales que involucran a grupos de las partes interesadas (Idem; Aliaga \& Luna, 2020).

La implementación de cursos y programas específicos sobre sustentabilidad mejoraría la alfabetización en cuestión. Por lo tanto, la renovación de los planes de estudio y de los métodos de aprendizaje se considera clave en la transición hacia la sustentabilidad. La idea de crear un plan de acción para fomentar la sustentabilidad y alfabetización ambiental es un evento global seguido por más de 500 Universidades de alrededor de sesenta países, en los que se encuentran Estados Unidos, Brasil, Taiwán, Canadá, Colombia, India o Australia como ejemplos representativos. Estas acciones incluyen la transformación de sus propias misiones, la inclusión de conceptos de desarrollo sustentable en sus planes de estudio, modificación de sus programas de investigación, introducción de nuevas formas de vida en sus campus, promoción de compromiso y evaluación de la comunidad. Sin embargo, la implementación holística de todas las partes sigue siendo un desafío (Disterheft, et al., 2013; Yáñez, 2018).

Los enfoques multidisciplinarios se consideran críticos para la sustentabilidad porque permiten adecuarse a una amplia variedad de perspectivas y soluciones. Investigadores que trabajan en proyectos multidisciplinarios a menudo reúnen experiencia y conocimiento de una variedad de disciplinas y partes interesadas para encontrar soluciones prácticas. Por esta razón argumentan que este enfoque es esencial para apoyar a la transición. 
En particular las Instituciones educación superior deben promover la reorientación de los planes de estudio hacia un desarrollo sostenible como una forma viable de aprender a identificar y formular metas importantes considerando una sociedad más justa alineada a las dimensiones económicas y ambientales (Makrakis y Kostoulas, 2013; Berchin, et al., 2018).

La transformación a una Institución sustentable tiene ciertas barreras especiales, como la libertad académica, la estructura activa y la administración conservadora que crean desafíos en el sentido de que la promoción de la sustentabilidad no se puede imponer. Mientras tanto Roorda enfatiza una estrategia de integración donde compara una licenciatura o un programa con un árbol, sus partes y su entorno educando y capacitando a los estudiantes a desarrollar continuamente niveles de competencias tanto personales como laborales. Durante el proceso de integración del desarrollo sustentable se fortalece la conciencia y participación de los estudiantes a reconocer sus responsabilidades ante la sociedad y el medio ambiente. Las Universidades deben estar comprometidas con actividades creíbles y prácticas para transmitir y mejorar el desempeño de sustentabilidad que beneficien la reducción de impactos ambientales (Roorda, 2013; Yáñez et al., 2018; Mendoza et al., 2019).

\section{Gestión del proceso hacia la sustentabilidad}

El cambio a la sustentabilidad sigue aspectos de la organización que pocos estudios se centraron en estudiarlo. El proceso para liderar la gestión del cambio que fue estudiado por Yáñez se ha utilizado en esta sección para el análisis en la implementación de sustentabilidad en la educación superior. El proceso consiste en crear un sentido de urgencia. Esto requiere persuadir al público de la necesidad de un cambio. Crear un cambio es un proceso delicado que requiere la participación de una masa crítica de personas dentro de las Universidades que deben adoptar el concepto de sustentabilidad y la necesidad de implementarlo. Para esto es importante formar estudiantes líderes que guíen el proceso del cambio mediante técnicas estructuradas. Estas técnicas están relacionadas a la comunicación (Loc. Cit.).
En el contexto de desarrollo sustentable es fundamental haber adquirido un alto grado de habilidad para escuchar, comunicar, construir relaciones, desarrollar la visión, ser receptivo y de adaptarse a una estrategia continua. También las Universidades trabajan en rigurosos procesos de carácter integrador con el propósito de cumplir los objetivos de formación para establecer y conservar un desempeño profesional eficaz y eficiente. Es esencial crear metas que contemplen los problemas a los que se enfrentará la organización e intentar prevenirlos. Los procesos de gestión del cambio toman tiempo, y las políticas de incentivos pueden ser rígidas. Por lo tanto, los agentes a menudo deberán estar motivados solamente por los efectos positivos del cambio. Por último, es necesario anclar la cultura del cambio en los planes estratégicos dentro del proceso sustentable como un ciclo de mejora continua. (Idem; Tapia et al., 2020).

\section{Vinculación de Ingeniería en la Industria}

La formación profesional del futuro debe ser capaz de armonizar los saberes pragmáticos con los conocimientos teóricos. Los procesos de transformación en la educación enfrentan como uno de los principales retos dar respuesta de forma más responsable a las demandas de los sectores productivos y sociales. Como consecuencia de establecer las estrategias alternativas para el futuro en un marco de desarrollo sustentable los desafíos de la realidad del siglo XXI requieren de la cooperación y la solidaridad como los nuevos fundamentos para evitar la marginación y fragmentación del conocimiento (Chávez, 2014).

Se mencionan a continuación dos criterios que figuran fuertemente la relación con la sustentabilidad y el desarrollo sustentable. El primero es la capacidad de aplicar el diseño de ingeniería a producir soluciones que satisfagan necesidades específicas. Por lo tanto, el pensamiento del diseño es imprescindible en la asignación de tareas creativas y complejas para superar Industrias cuyos procesos de diseño son rudimentarios. Esto apoya a una manufactura comprometida con la biodiversidad, el cambio climático, reducción de la pobreza, paz y seguridad humana. Sin embargo, el aprendizaje debe centrarse en la calidad de las habilidades claves de los estudiantes y su relación con la experiencia de las Industrias (Katz, 2015; Sengupta, 2017; Thurer et al., 2018). 
El segundo se refiere a la capacidad de reconocer las responsabilidades éticas y profesionales y hacer juicios informados que deben considerar el impacto de las soluciones de ingeniería (Katz, 2015; Sengupta, 2017; Thurer et al., 2018).

Las empresas juegan un papel importante como difusores de negocios innovadores en economía circular pero no son suficiente para impulsar el cambio requerido. Las innovaciones se refieren al proceso de diseños de más allá del individuo y prácticas tradicionales con el propósito de guiar la transformación corporativa y mejorar la ventaja competitiva. La economía circular aumenta la capacidad de una organización para anticipar, prepararse, responder y adaptarse a situaciones radicales e interrupciones repentinas para sobrevivir y prosperar sobrepasando barreras relevantes. La formación profesional del futuro debe centrarse en la enseñanza de los valores humanos de manera que desaliente el individualismo egoísta, que promueva el respeto a la vida en la sociedad, sin ignorar la solidaridad social, la conservación de la naturaleza y el medio ambiente (Mendoza et al., 2019; Vargas, 2020).

Lograr la consolidación de las partes implica plantear el cambio de programas de estudio, sus tiempos, los métodos de enseñanza y técnicas, así como la integración de contenidos mismos de la disciplina. La vinculación del Estado en conjunto con las Universidades, empresas y la sociedad es esencial para complementar los esfuerzos que plantea el crecimiento demográfico, de tal forma que se puedan crear y desarrollar profesiones necesarias a la realidad económica, social y política. La vinculación de la educación con los sectores productivos y sociales tendrá que fortalecer el desarrollo de proyectos con responsabilidad compartida. Este vínculo es uno de los principales desafíos para los países en desarrollo. Es por ello que se necesita una Educación Superior estructurada que garantice programas de estudio de calidad sin importar el nivel o prestigio de las Universidades en México (Mahmud et al., 2012; Chávez, 2014; Espinosa et al., 2020).
La naturaleza de la educación superior permite a las Universidades enseñar y preparar a futuros profesionales a desarrollar nuevas herramientas para enfrentar nuevos desafíos en la sociedad. Debido a sus estructuras y recursos de investigación pueden compartir su conocimiento para aplicar nuevos modelos sociales en la gestión para mantener la coherencia a la hora de aportar valor a las empresas y a la sociedad. A propósito de la idea de diseñar un producto que su vida ecológica sea consumir y desechar, se pretende reutilizar o crear un nuevo valor después de utilizarlo. Sin embargo, una transformación de los sistemas de cadena de valor a ese nivel requiere adaptarse a otro estilo de producción y consumo (Idem; Yáñez et al., 2018).

\section{Metodología}

Esta investigación tuvo un enfoque cualitativo para interpretar de manera flexible y compleja un conjunto de sistemas a partir observaciones no estructuradas basadas en un proceso inductivo con el propósito de explorar, describir y generar perspectivas teóricas detalladas. De una revisión inicial de publicaciones de carácter científico y académico (por mencionar la plataforma digital ScienceDirec), se perfeccionó el seguimiento de 28 artículos orientados a una educación superior sustentable para el planteamiento del problema. El enfoque cualitativo permitió plantear el problema sin seguir un proceso claramente definido con planteamientos no específicos. Se examinó la cuestión sobre mejorar el entorno del mundo y en este proceso se desarrolló una teoría coherente de acuerdo con lo que se observó con el propósito de reconstruir la realidad siguiendo un método holístico.

\section{Resultados}

Como punto de partida se sugiere la implementación de actualizaciones y mejoras en los programas de estudio en las facultades de Ingeniería con la finalidad de orientar a los estudiantes a un desarrollo sustentable que abarque aspectos socioeconómicos y ambientales. Cabe mencionar que se encontraron diversas estrategias de integración que buscan una mejora en el sistema educativo que van desde pequeñas modificaciones en planes de estudio hasta una reestructuración compleja en las Universidades. 
Está claro que la vinculación de la Ingeniería con la Industria ilustra el compromiso de responder a las necesidades del mercado del consumismo, es por ello que se enfatiza como prioridad trabajar en conjunto dando respuestas a problemas socioeconómicos mediante diseños de productos y procesos que respeten el medio ambiente.

\section{Conclusiones}

El objetivo de esta investigación fue estudiar a las Universidades que están implementando nuevos modelos educativos prestando atención a las facultades de Ingeniería. Se llegó a la conclusión de que es necesario guiar a las futuras generaciones hacia un camino sustentable capaces de comprender fenómenos naturales, sociales y económicos. No solo deben observar los cambios que vayan surgiendo, sino que deben analizarlos de forma crítica y exhaustiva para proponer soluciones o mejoras innovadoras. Para que esto suceda se planteó que es fundamental el trabajo en conjunto con otras disciplinas para tener así un panorama más amplio de todo lo que esté sucediendo. Por ejemplo, en la correlación con la disciplina de Filosofía y Letras realizarán trabajos de colaboración para la realización de reportes escritos que fomente a los estudiantes de Ingeniería a expresar sus ideas de forma clara. Por otro lado, se correlaciona con la disciplina de Ciencias de la Comunicación en tiempo real, logrando negociaciones a distancia involucrando también áreas administrativas como Economía, Contabilidad, Negocios Internacionales formando ingenieros integrales preparados para enfrentar el entorno actual de acuerdo con los objetivos de los representantes de los empleadores del sector ingenieril que buscan profesionistas y profesionales para resolver sus problemas. En los últimos años muchas Universidades comenzaron a adoptar acciones dirigidas hacia un desarrollo que contemple a los individuos, Instituciones, el diseño de productos y estructuras relacionado con la opción de un desarrollo eficiente y rentable.

De tal manera que se consiga el equilibrio y que todas las partes queden satisfechas. Se tomó en cuenta que las Industrias son los principales elementos en los que las Universidades pueden obtener soporte y trabajar mutuamente para desarrollar proyectos que conlleven a nuevos diseños que proporcionen beneficios para ambas partes.

\section{Referencias}

Abdul, K. M. (2016). Entrepreneurship Education In An Engineering Curriculum. Procedia Economics and Finance, 35, 379-387. https://doi.org/10.1016/S2212-5671(16)000472

Aliaga, A. A., \& Luna, J. (2020). La construcción de competencias investigativas del docente de posgrado para lograr el desarrollo social sostenible. Revista ESPACIOS. ISSN, 798, 1015.

Berchin, I. I., Sima, M., Andrade De L. M., Biesel, S., Dos Santos, L. P. Ferreira, R. V... \& Ceci, F. (2018). The importance of international conferences on sustainable development as higher education institutions' strategies to promote sustainability: A case study in Brazil. Journal of Cleaner Production, 171, 756-772. https://doi.org/10.1016/j.jclepro.2017.10.042

Chávez, J. (2014). La educación en México bajo el horizonte del año 2020. México. Revista Iberoamericana de Producción Académica y Gestión Educativa, 1, 1-20

Chowdhury, H., Alam, F., Biswas, S. K., Islamb, M. T. \& Islamc, S. (2013). Quality assurance and accreditation of engineering education in Bangladesh. Procedia Engineering, $\quad 56, \quad 864-869$. https://doi.org/10.1016/j.proeng.2013.03.208

Derrick, S. (2013). Time and Sustainability Metrics in Higher Education. Springer, 47-63. https://doi.org/10.1007/978-3-319-02375-5_3

Disterheft, A., Caeiro, S., Azeiteiro, U. M. \& Filho, W. L. (2013). Sustainability Science and Education for Sustainable Development in Universities: A Way for Transition. Springer, 3-27. https://doi.org/10.1007/978-3-319-023755_1

Espinosa, A., Carvajal, A. L., Romero, C., Rivera, C. E., Peralta, F., Alcívar, F., ... \& Castelo, J. F. (2020). Nuevas Tendencias en Educación, Ciencia, Tecnología y Sociedad.

Glover, A. \& Peters C. A. (2013). Whole Sector Approach: Education for Sustainable Development and Global Citizenship in Wales. Springer, 205-222. https://doi.org/10.1007/9783-319-02375-5_12

MARCOS-SORIANO, José Antonio, CRUZ-GÓMEZ, Marco Antonio, MEJÍA-PÉREZ, José Alfredo y ESPINOSACARRASCO, María del Rosario. La tendencia del desarrollo sustentable en la educación en ingeniería en México. Revista del Desarrollo Urbano y Sustentable. 2020 
Katz, R. (2015). Integrating Analysis and Design in Mechanical Engineering Education. Procedia CIRP, 36, 23-28. https://doi.org/10.1016/j.procir.2015.01.042

Lozano, R. \& Watson, M. K. (2013). Assessing Sustainability in University curricula: Case Studies from the University of Leeds and the Georgia Institute of Technology. Springer, 359373. https://doi.org/10.1007/978-3-319-023755_20

Mahmud, J.O., Ismail, M. \& Taib, J. M. (2012). Engineering Education and Product Design: Nigeria's Challenge. Procedia - Social and Behavioral Sciences, 56, 679-684. https://doi.org/10.1016/j.sbspro.2012.09.703

Makrakis V. \& Kostoulas, N. M. (2013). A Methodology for Reorienting University Curricula to Address Sustainability: The RUCAS-Tempus Project Initiative. Springer, 323-344. https://doi.org/10.1007/978-3-31902375-5_18

Mendoza, J., Gallego, A. \& Azapagic, A. (2019). Building a business case for implementation of a

circular economy in higher education institutions. Journal of Cleaner Production, 220 , 553-567. https://doi.org/10.1016/j.jclepro.2019.02.045

Momete, D. C. (2015). Fostering innovation and entrepreneurial culture in Romania through engineering higher education programs. Procedia Economics and Finance, 22, 168 $174 . \quad$ https://doi.org/10.1016/S22125671(15)00253-1

Mujumdar, A. S. (2014). Editorial: Higher Education in Engineering: Need for Paradigm Shift. Drying Technology, 32(12), 1397. https://doi.org/10.1080/07373937.2014.928063

Mulder, K. F. (2017). Strategic competences for concrete action towards sustainability: An oxymoron? Engineering education for a sustainable future. Renewable and Sustainable Energy Reviews, 68, (2), 1106-1111. https://doi.org/10.1016/j.rser.2016.03.038
Mulder, K.F., Ferrer, B.D., Segalas, C.J., Kordas, O., Nikiforovich, E. \& Pereverza, K. (2013). Being Scared is not Enough! Motivators for Education for Sustainable Development. Springer, 29-45. https://doi.org/10.1007/978-3319-02375-5_2

Ortega, M. D. L. Á. M. (2020). La educación como fundamento orientador hacia una cultura ambiental. RIDE Revista Iberoamericana para la Investigación y el Desarrollo Educativo, 10(20).

Pappas, E., Pierrakos, O. \& Nagel, R. (2013). Using Bloom's Taxonomy to teach sustainability in multiple contexts. Journal of Cleaner Production, 48, 54-64.

https://doi.org/10.1016/j.jclepro.2012.09.039

Roorda, N. (2013). A Strategy and a Toolkit to Realice System Integration of Sustainable Development (SISD). Springer, 101-119. https://doi.org/10.1007/978-3-319-02375-5_6

Sengupta, D., Huang, Y., Davidson, C.I., Edgar, T.F., Eden, M.R. \& El-Halwagi, M.M. (2017), Using module-based learning methods to introduce sustainable manufacturing in engineering curriculum. International Journal of Sustainability in Higher Education, 18, (3),307-328, https://doi.org/10.1108/IJSHE-052015-0100

Sonmez, M. (2014). The Role of Technology Faculties in Engineering Education. Procedia Social and Behavioral Sciences 141, 35 - 44. https://doi.org/10.1016/j.sbspro.2014.05.009

Staniskis, J. K. \& Katiliute, E. (2015). Complex evaluation of sustainability in engineering education: case \& análisis. Journal of Cleaner Production, 120, 13-20.

https://doi.org/10.1016/j.jclepro.2015.09.086

Tapia, I. I., Moreno, M. R., \& Marrero, C.E. (2020). La gestión en el colectivo del año académico universitario, Revista de Desarrollo Sustentable, Negocios, Emprendimiento y Educación RILCO DS, 2, (3), 1-14.

https://www.eumed.net/rev/rilcoDS/03/universi dades-cubanas.html http://hdl.handle.net/20.500.11763/rilcoDS03un iversidades-cubanas 
Thurer, M., Tomasevic, I., Stevenson, M., Qu, T. \& Huisingh, D. (2018). A systematic review of the literature on integrating sustainability into engineering curricula. Journal of Cleaner Production, 181, 608-617.

https://doi.org/10.1016/j.jclepro.2017.12.130

Vargas, F. (2020). La Ciudad Región Global para el desarrollo económico y social de Latinoamérica. Aibi revista de investigación, administración e ingeniería, 58-68.

Yáñez, S. Uruburu, A., Moreno, A. \& Julio, L. (2018). The sustainability report as an essential tool for the holistic and strategic vision of higher education institutions. Journal of Cleaner Production, 207, 57-66.

https://doi.org/10.1016/j.jclepro.2018.09.171. 